\title{
Fabrication of Micro- and Submicrometer Period Metal Reflection Gratings by Melt-Imprint Technique
}

\author{
Bálint KISS $^{* 1}$, Roland FLENDER ${ }^{* 1}$ and Csaba VASS ${ }^{* 1}$ \\ ${ }^{* 1}$ Department of Optics and Quantum Electronics, University of Szeged, H-6720 Szeged, Dóm tér 9, \\ Hungary \\ E-mail: vasscsaba@physx.u-szeged.hu
}

\begin{abstract}
In this study we report on a two step method to produce reflection gratings (period: from $266 \mathrm{~nm}$ to $3710 \mathrm{~nm}$ ) in bulk tin (Sn). In our first step, surface relief gratings were etched into fused silica plates by two-beam interferometric laser-induced backside wet etching (TWIN-LIBWE) technique based on a nanosecond Q-switched frequency-quadrupled Nd:YAG laser. In the second step the grooved structures were copied into bulk tin by melting and imprinting of tin target. Both the fused silica masters and tin replicas were characterized by atomic force microscopy (AFM). The reachable modulation depths of tin replicas were found to be near constant at each period.
\end{abstract}

DOI: 10.2961/jlmn.2013.03.0016

Keywords: Metal gratings, imprint technique, TWIN-LIBWE, submicrometer resolution

\section{Introduction}

Optical gratings are fundamental elements of optics, spectroscopic devices and arrangements. Especially the high damage threshold reflection gratings are essential in high intensity laser systems. The gratings in bulk metal substrates have higher damage threshold than the nowadays used reflection gratings (based on polymer structure covered by metal film), since the good heat diffusivity of the bulk metals can help to conduct the absorbed laser energy from the irradiated area without damaging of grating.

The laser material processing is a powerful tool to microstructure surfaces [1]. The laser based production of periodic structures in bulk metals is primary limited by the optical resolution: the minimum structure size is proportional to the illuminating wavelength. Upon ablation the reachable minimum period is also limited by the laser pulse duration: the significant heat diffusion [2] along the surface can destroy the (sub)micrometer size structures in case of (long) ns pulses. UV pulses provide sufficient resolution for the most commonly desired grating periods (some hundred nanometers).

In this study we report on a two step method based on the combination of indirect laser-based technique and a melt-imprint procedure. First, master gratings were fabricated in fused silica by laser-induced backside wet etching (LIBWE), then the surface relief structures were transferred in bulk tin ( $\mathrm{Sn})$.

The fused silica is an ideal master grating material, due to its high melting point, chemical and physical stability and mechanical hardness. For microstructure of fused quartz, the laser-induced backside wet etching (LIBWE) technique $[3,4]$ is proved to be a flexible and effective tool. LIBWE can be applied e.g. for the fabrication of microoptical elements [5,6] and various structures for biological, or biotechnological use [7]. High quality, high resolution gratings can be fabricated by two-beam interferometric (TWIN-) LIBWE technique [8-10]: the reachable minimal period of the structure is near $100 \mathrm{~nm}$ [8]. In many applica- tion, where the resolution of the structures is high enough, and the smoothness of surface is also sufficient, the relatively simple and low-cost TWIN-LIBWE technique can be good alternative beside and instead of the complicated and expensive reactive ion techniques [11].

Besides laser based methods, there are numerous approaches to realize micro- and nanoscale patterns in order to produce low-scale devices for various applications from microoptics- and electronics to microfluidics [12-19]. From these creative methods the most relevant technique for our purpose is the imprint lithography which generally designed to replicate the surface structures of a master element [20-23]. This method is simple, flexible, costeffective and productive, while the maximal resolution is around ten nanometers.

Our aim is to demonstrate that metal grating can be fabricated by the combination of two simple and cost effective techniques: LIBWE and melt-imprint lithography.

\section{Experimental}

The imprinted tin gratings were produced in two steps. In the first step, surface relief gratings were etched into fused silica plates by two-beam interferometric laserinduced backside wet etching (TWIN-LIBWE) technique based on a Q-switched frequency-quadrupled Nd:YAG laser source $\left(\lambda=266 \mathrm{~nm}, \tau_{F W H M}=8 \mathrm{~ns}\right.$, repetition rate: $\left.10 \mathrm{~Hz}\right)$ $[8,9]$. The experimental setup was detailed in our previous studies $[8,9]$ and the scheme is plotted on Fig.1. 


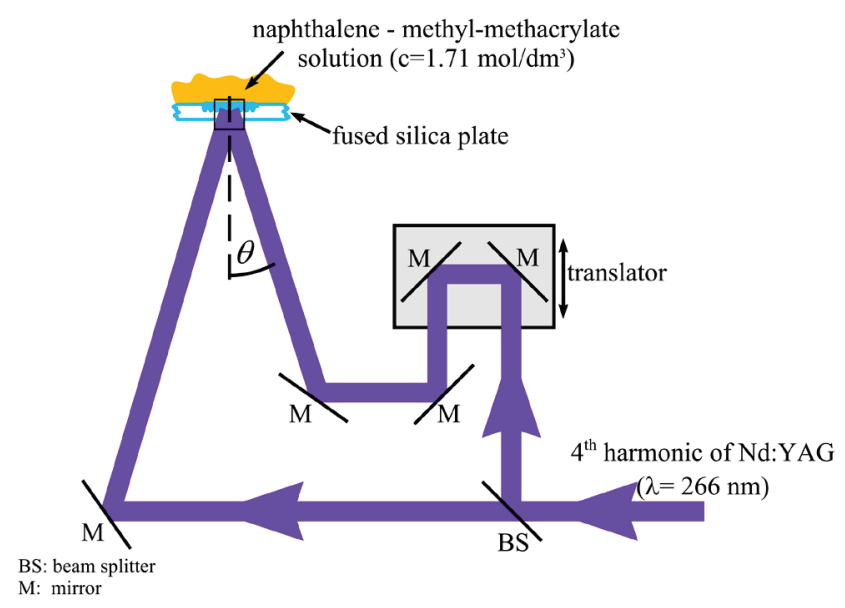

Fig. 1 Scheme of the experimental setup for fused silica (master) grating fabrication

The number of laser pulses was varied between 10 and 150; the laser fluence was altered between 235 and 400 $\mathrm{mJ} / \mathrm{cm}^{2}$. The liquid absorber was saturated solution of naphthalene and methyl-methacrylate $\left(\mathrm{c}=1.71 \mathrm{~mol} / \mathrm{dm}^{3}\right)$. The grating period, " $p$ " $(266,505,1045,2120$ and 3710 $\mathrm{nm})$ were adjusted by the incident angles $\left(\theta=30^{\circ}, 15,3^{\circ}\right.$, $\left.7,3^{\circ}, 3,6^{\circ}, 2,05^{\circ}\right)$ of laser beams. The same experimental setup (Fig.1) was used for the direct ablation of tin. The sample was changed to a tin plate. After the fine adjustment of the setup, the period $(505,1055,2115$ and 3710 $\mathrm{nm}$ ) was controlled also by the incident angle of the laser beams. The applied fluence was $330-600 \mathrm{~mJ} / \mathrm{cm}^{2}$, the number of laser pulses were varied from 1 to 20 .

In the second step the grooved fused silica structures were copied into bulk tin. Upon the imprinting both the masters and the pieces of high purity tin were heated up above the melting temperature of $\mathrm{Sn}\left(\mathrm{T}_{\text {Sn melting point }} \approx 230^{\circ} \mathrm{C}\right)$ by conventional heat source, in ambient air and at normal pressure. After the removal of the oxidized Sn layer the clean, melted tin was placed onto the master's grooved surface (Fig.2.). After an approx. 5 minutes cool down period, the resolidified tin with the negative periodic profile of master's imprinted on its surface was removed from the master grating.

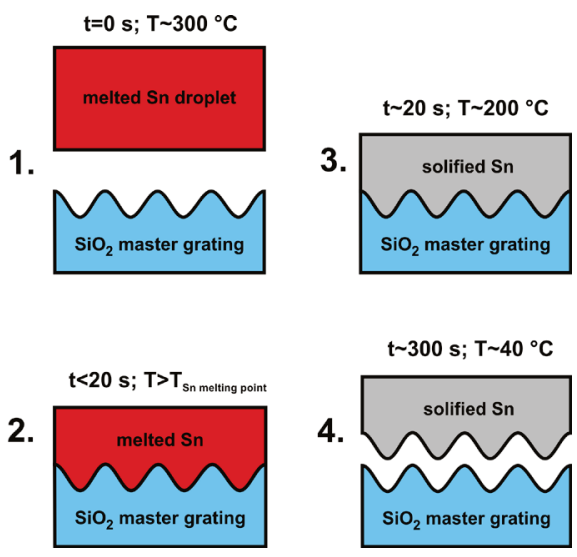

Fig. 2 Schematic of the melt-imprint method for reflection grating fabrication in bulk tin
Without heating up of master substrate the significant thermal expansion of fused silica plate causes the cracking of master substrates during the cool down period. The reduction of the size of master plates is also important to prevent the cracking of master plates. After the wet etching procedure, the $1 \times 1$ inch size quartz plates were cut into smaller pieces of $0.5 \times 0.5$ inch parts. With these methods the cracking was successfully prevented and the masters can be used again. If the tin stuck on the master, it could be completely removed by hydrochloric acid in ultrasonic bath.

The surface morphology of the gratings (fused silica masters and tin replicas) was studied and their modulation depths (MD) were measured by PSIA XE-100 atomic force microscope (AFM).

\section{Results and discussion}

\subsection{Direct ablation of tin}

In our first experiments, an attempt were made to produce gratings into bulk tin with our experimental setup (Fig.3.) based on a nanosecond laser. Gratings can be observed on the ablated tin surface only in case of the highest period (3710 nm) with fluence just above the ablation threshold of $\operatorname{tin}\left(\approx 380 \mathrm{~mJ} / \mathrm{cm}^{2}\right)$, while the pulse number has to be strictly one. The second pulse destroys the structures see in the AFM image (Fig.3b), due the high heat diffusivity of tin. The thermal diffusion length $\left(l_{T}=2 \sqrt{D \tau}\right.$, where $D$ is heat diffusivity, and $\tau$ is laser beam dwell time [1]) was calculated for tin: it was found to be near $1 \mu \mathrm{m}$, which is in the magnitude of the minimum grating period (4 $\mu \mathrm{m})$.
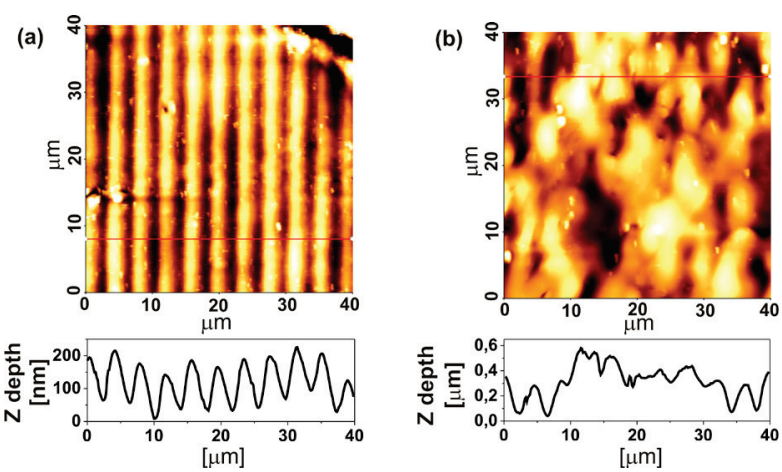

Fig. 3 AFM images of direct ablated bulk tin grating period: $3710 \mathrm{~nm}$; laser fluence: $380 \mathrm{~mJ} / \mathrm{cm}^{2}$

(a) 1 pulse; (b) 2 pulses

Decreasing the period ( $p: 2115,1055,505 \mathrm{~nm})$, the grooves can not be observed at any fluence values independently of pulse quantity after interferometric direct ablation. We proved with these experiments that normal nanosecond laser is not suitable for grating fabrication in bulk tin with period smaller than $\approx 4 \mu \mathrm{m}$.

\subsection{Melt-Imprinting of tin}

The laser fluence and number of pulses were adjusted at each period to cover as high modulation depth range of fused silica master as possible. For an example a photo about a whole tin replica can be seen in Fig. 4 ( $p=1045 \mathrm{~nm})$ 


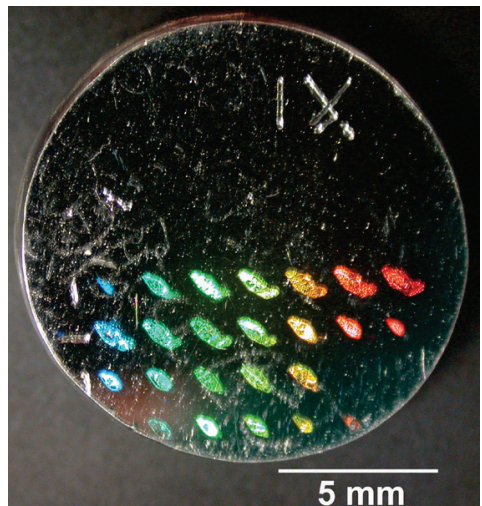

Fig. 4 Photo about a tin replica $(\mathrm{p}=1045 \mathrm{~nm})$ The parameters of master: constant fluence values in each rows $\left(400-290 \mathrm{~mJ} / \mathrm{cm}^{2}\right)$ and series of pulse number (10-150 pulses)

We started the imprinting experiments and analysis with the highest period gratings. The AFM images of a master fused silica grating and its replica in the case of $3710 \mathrm{~nm}$ period can be seen in Fig.5 (a) and (b). The dependence of modulation depth of tin replicas on the modulation depth of master fused silica grating was plotted on Fig. 5.c. While at this period the surface profiles of master fused silica grating are perfect, the peaks of the tin grooves are broken in many cases, causing modulation depth decrease and quality loss of tin replicas. According to the Fig.5.c., the modulation depth of tin replicas is independent from the MD of master fused silica gratings. The reachable $\mathrm{MD}$ of tin structures in case of $3710 \mathrm{~nm}$ master period can be calculated as the average MD of tin structures, which was found to be $122 \mathrm{~nm}$.
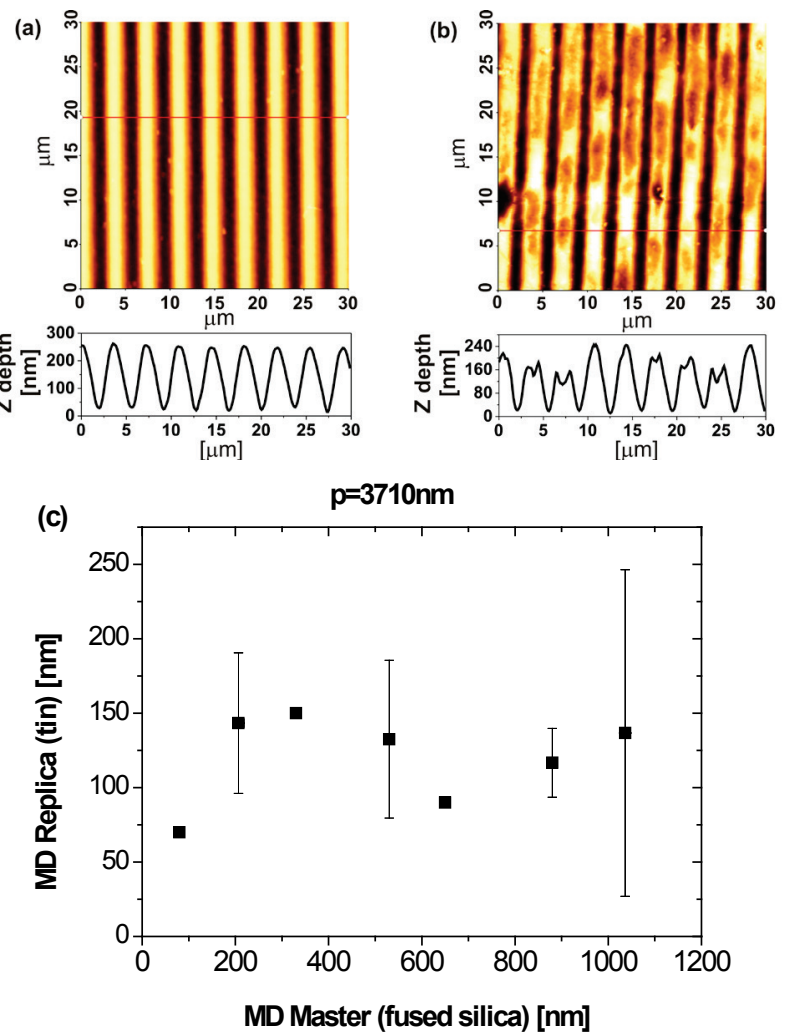

Fig. 5 AFM images of master fused silica grating (a) and its tin replica (b), and

the MD tin vs. MD fused silica (c); $p=3710 \mathrm{~nm}$
In our further experiments the incident angles were increased in order to decrease the period of the masters. The optimal laser parameter set becomes narrower with decreasing grating constant [8]. The illustration of results are similar to the previous case: an AFM image of a master fused silica grating and its replica can be seen in figures 69 (a) and (b), respectively, while the dependence of MD of tin replicas on the MD of master fused silica grating was plotted on figure 6-9 (c). The maximum MD and the quality of fused silica gratings at each period decrease with decreasing of the grating period [8-10]. This behaviour possibly originates from (i) the decreasing of the visibility of the interference pattern and (ii) the thermal effects $[2,8,9]$.
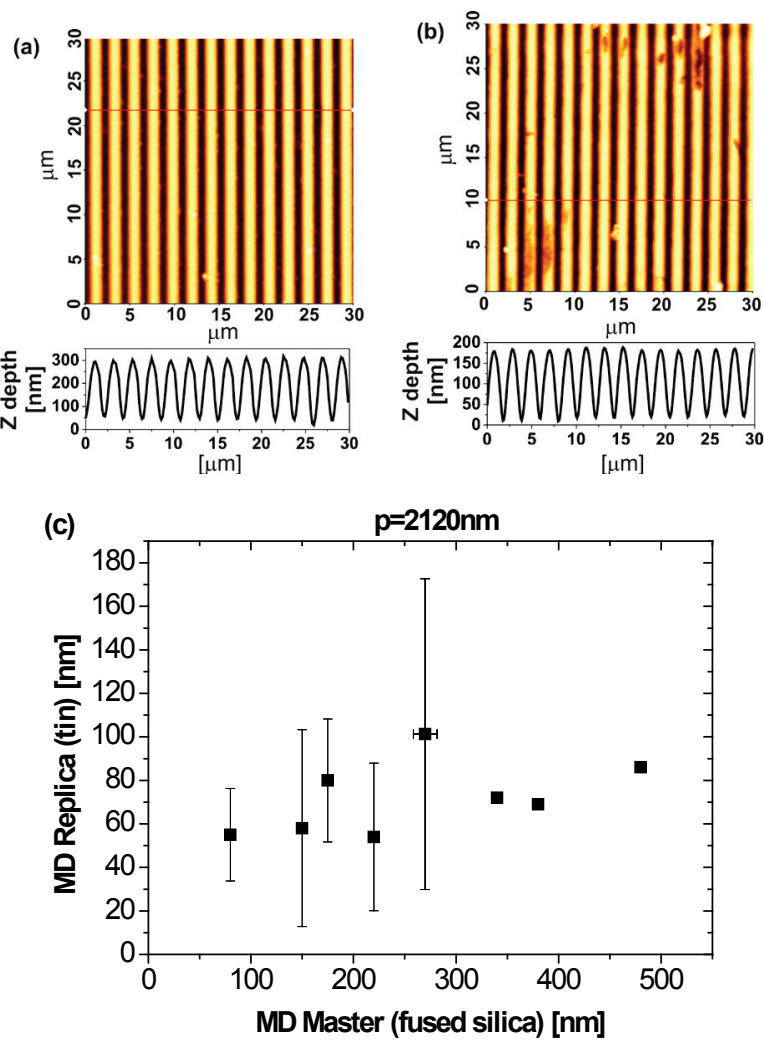

Fig. 6 AFM images of master fused silica grating (a) and its tin replica (b) and

the MD tin vs. MD fused silica (c); $p=2120 \mathrm{~nm}$ 

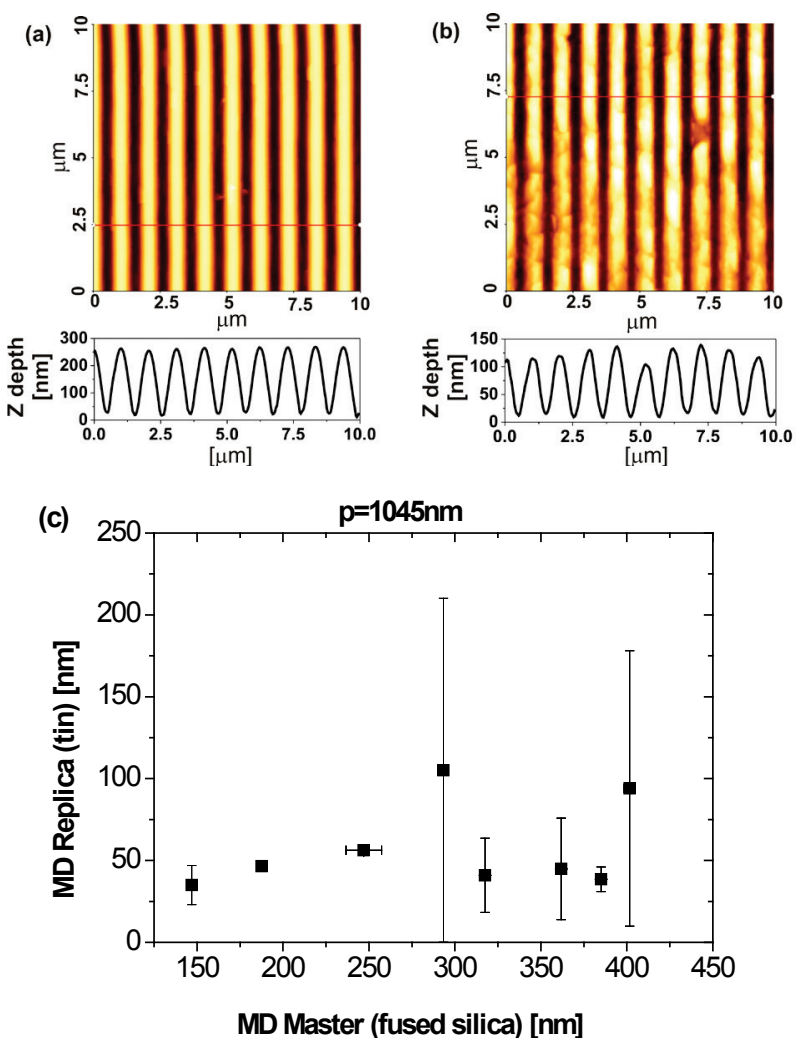

Fig. 7 AFM images of master fused silica grating (a) and its tin replica (b) and

the MD tin vs. MD fused silica (c); $p=1045 \mathrm{~nm}$

(b) image corresponds to spot on Fig.4 ( $3^{\text {rd }}$ row, $2^{\text {nd }}$ column)

Laser parameters of master: $310 \mathrm{~mJ} / \mathrm{cm}^{2} ; 20$ pulses
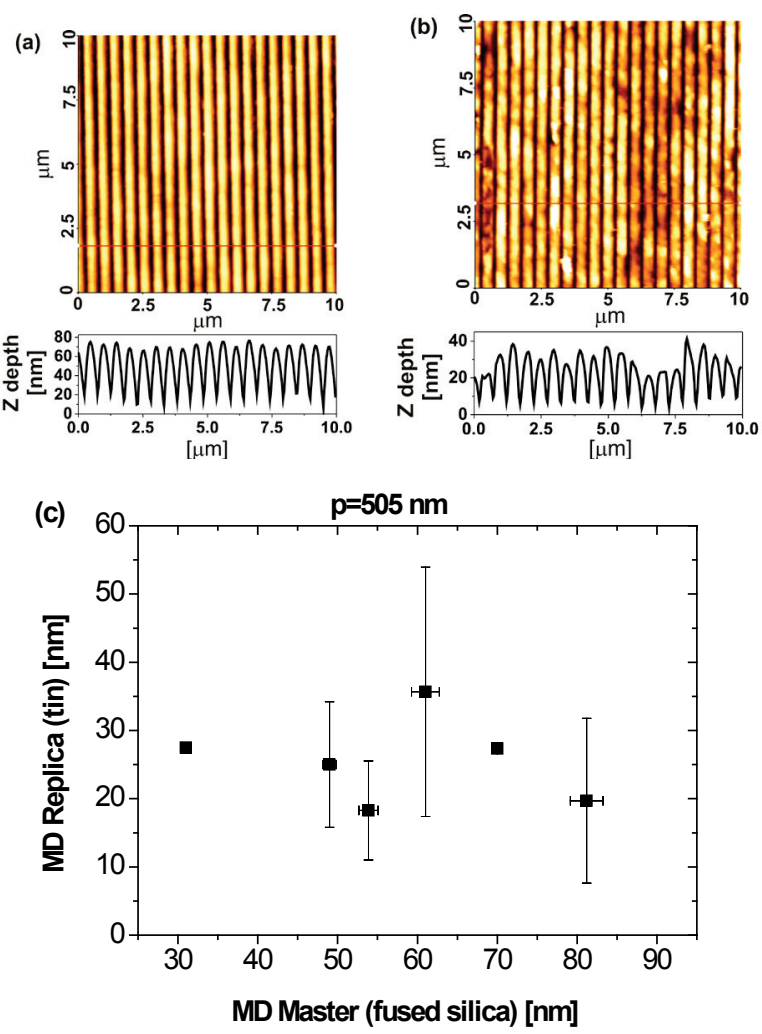

Fig. 8 AFM images of master fused silica grating (a) and its tin replica (b) and

the MD tin vs. MD fused silica (c); $p=505 \mathrm{~nm}$
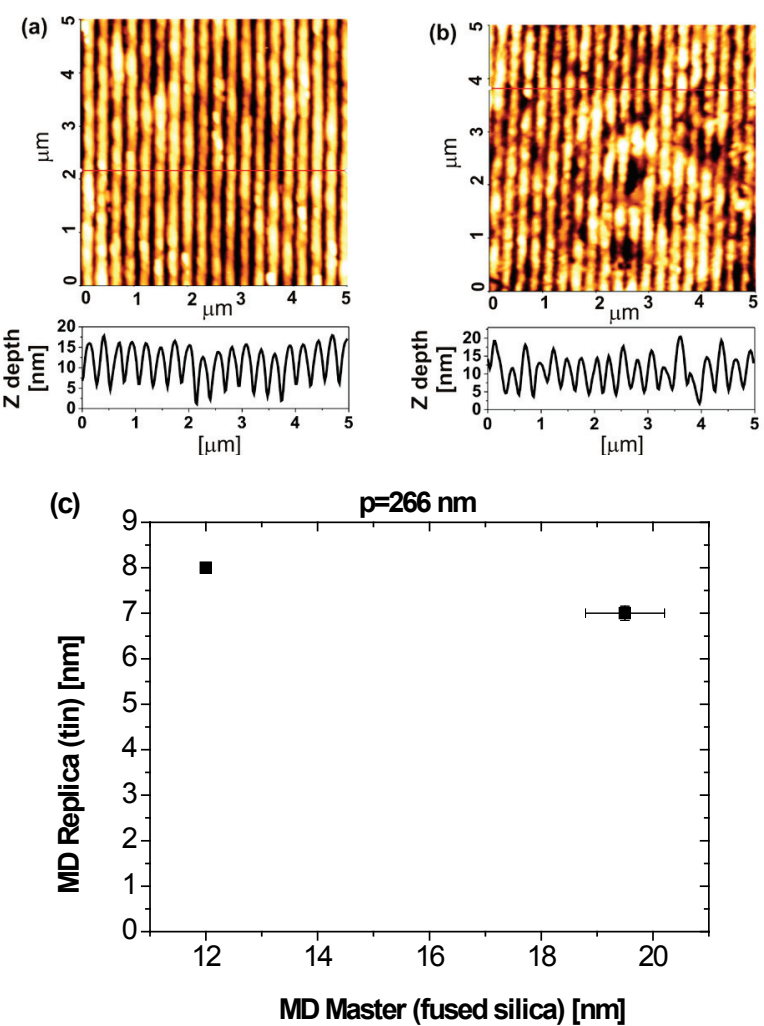

Fig. 9 AFM images of master fused silica grating (a) and its tin replica (b) and

the MD tin vs. MD fused silica (c); $p=266 \mathrm{~nm}$

The broken grooves can be observed in the last four cases ( $p=2120-266 \mathrm{~nm}$; Fig. 6-9), but the area of this deteriorated gratings seem to be smaller than in largest period case, although the correct comparison is difficult due to the degradation of master gratings with the decreasing of period. The modulation depths of tin replicas are independent from the modulation depths of master fused silica gratings for each period. The dependence of average modulation depths of tin structures on the master period was plotted in Fig. 10.: this graph demonstrates the limit of our two-step technique.

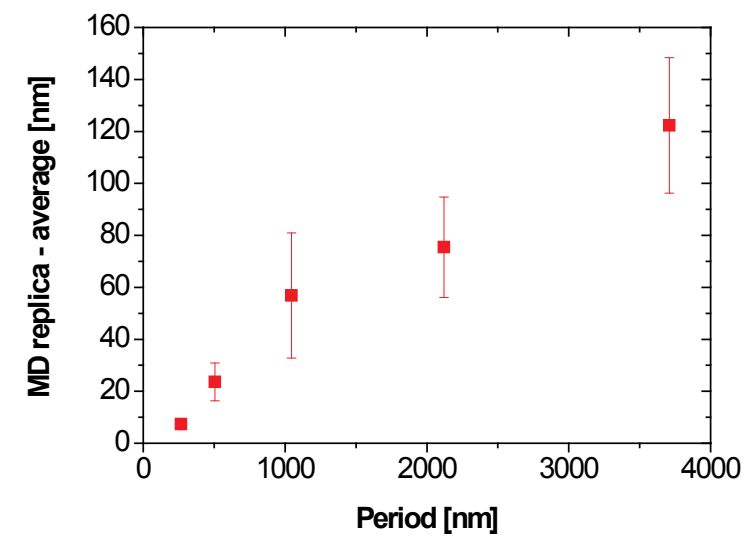

Fig. 10 Average modulation depth of tin replicas vs. grating period 
The formation of tin grating during the melt-imprint procedure is rather complex. In our imprint method the tin is molten for about 20 seconds in contact with the mould opposite to the previously presented study on LADI [21], where the target is in liquid form for less than $250 \mathrm{~ns}$. Therefore the time dependence of melting and the effect of viscous force is not relevant. However, the wettability of fused silica grating by the melted tin plays key role in the tin replica origination. Furthermore the ambient air surrounding and its consequences (e.g. oxidation), and probably the air inclusion between the surfaces of fused silica grating and of melted tin can also be important during the melt-imprint procedure. The investigation and discussion of these effects are beyond the object of study, since our main aim was to demonstrate the suitability of our simple two-step technique for (sub)micrometer period metal grating fabrication.

In referred studies $[20,21]$ the structures were copied into metals by laser assisted direct imprinting technique (LADI), in which the (metal) target was heated by the absorbed laser energy under high pressure $(\approx 10 \mathrm{MPa})$. In contras with these, in our melt-imprint experiments heating laser source and pressing is unnecessary to produce the presented tin reflection gratings.

We demonstrated that the fabricated tin replicas can be applied as refection gratings. Photos about the diffracted orders of a HeNe laser beam from the tin replicas can be observed in the Fig.11. The small deviation of diffracted spots is originated from the non-perpendicular incident angle.

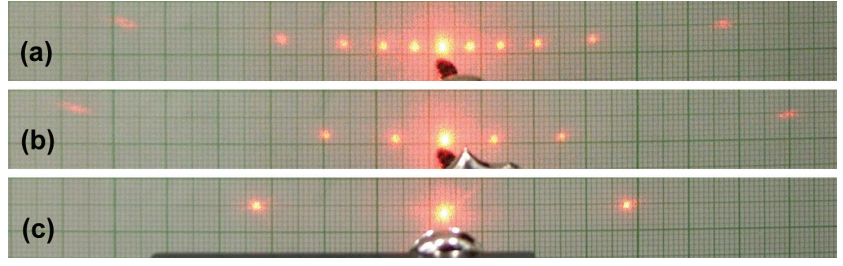

Fig. 11 Photo about the diffracted HeNe spots: (a) $p=3710 \mathrm{~nm}$; (b) $p=2120 \mathrm{~nm}$; (c) $p=1045 \mathrm{~nm}$

\section{Summary}

In this study we demonstrated that tin metal gratings can be fabricated by the combination of two simple and cost effective techniques: TWIN-LIBWE and melt-imprint lithography. The master gratings with periods from 3710 to $266 \mathrm{~nm}$ were produced in fused silica by TWIN-LIBWE technique. These structures were transferred into bulk tin by melt-imprint technique. The reachable modulation depth of tin replicas was near constant for each period. According to our preliminary measurements, these structures can be applied as reflection grating.

\section{Acknowledgments}

The authors gratefully acknowledge the financial support of Hungarian Scientific Research Fund (OTKA PD 104477). This research was supported by the European Union and the State of Hungary, co-financed by the European Social Fund in the framework of TÁMOP 4.2.4. A/211-1-2012-0001 'National Excellence Program. The project was subsidized by the European Union and co-financed by the European Social Fund. The project was partially funded by TÁMOP-4.2.2.A-11/1/KONV-2012-0060 - „Impulse lasers for use in materials science and biophotonics" is supported by the European Union and co-financed by the European Social Fund.

\section{References}

[1] D. Bäuerle, Laser Processing and Chemistry, 3rd ed., (Springer, Berlin, 2000)

[2] P. Simon and J. Ihlemann: Appl. Surf. Sci., 109/110, (1997) 25.

[3] J. Wang, H. Niino and A. Yabe: Appl. Phys. A 68, (1999) 111.

[4] S. I. Dolgaev, A. A. Lyalin, A. V. Simakin and G. A. Shafeev: Appl. Surf. Sci. 96-98, (1996) 491.

[5] G. Kopitkovas, T. Lippert, C. David, A. Wokaun and J. Gobrecht: Microel. Eng. 67-68, (2003) 438.

[6] K. Zimmer and R. Böhme: Appl. Surf. Sci. 243, (2005) 415.

[7] J.Y. Cheng, M.H. Yen, C.W. Wei, Y.C. Chuang and T.H. Young: J. Micromech. Microeng. 15, (2005) 1147

[8] Cs. Vass, K. Osvay, T. Véső and B. Hopp: Appl Phys A, 93, (2008) 69.

[9] Cs. Vass, K. Osvay and B. Hopp: Opt. Exp., Vol. 14, No. 18, (2006) 8354.

[10]Cs. Vass, K. Osvay, M. Csete and B. Hopp: Appl. Surf. Sci., 253, (2007) 8059

[11] X. Lia, T. Abeb and M. Esashi: Sensors and Actuators A, 87, (2001) 139.

[12] C. A. Mirkin and J. A. Rogers: MRS Bull., 26, (2001) 506.

[13] S.Y. Chou: MRS Bull., 26, (2001) 512.

[14] A. Braeuer, P. Dannberg, G. Mann and M. Popall: MRS Bull., 26, (2001) 519.

[15] J. C. Love, J. R. Anderson and G. M.Whitesides: MRS Bull., 26, (2001) 523.

[16] J. A. Rogers: MRS Bull., 26, (2001) 530.

[17] C. A. Mirkin: MRS Bull., 26, (2001) 535.

[18]H. Sirringhaus, T. Kawase and R.H. Friend: MRS Bull., 26, (2001) 539.

[19] K. Matsumaru and A. Takata: MRS Bull., 26, (2001) 544.

[20] S.Y. Chou, C. Keimel and J. Gu: Nature, 417, (2002) 835.

[21]B. Cui, C. Keimel and S. Y Chou: Nanotechnology 21, (2010) 045303

[22] J. Thomas, P. Gangopadhyay, E. Araci, R. A. Norwood and N. Peyghambarian: Adv. Mater., 23, (2011) 4782.

[23] S. Y. Chou, P. R. Krauss and P. J. Renstrom: Science, 272, (1996) 85.

(Received: August 19, 2013, Accepted: December 8, 2013) 